\title{
A Study of Puerperal Morbidities - In a Tertiary Care Centre
}

\author{
Dr. R C Prameela ${ }^{1 *}$, Dr. Smitha Ayli ${ }^{2}$ \\ ${ }^{1}$ 16th main, Vijayanagara 2nd stage Mysore, Karnataka 570017, India \\ ${ }_{2}^{2}$ Jayanagara 2nd stage, First cross, Gangavathi, Koppal district, Karnataka 583227, India
}

DOI: $10.36348 /$ sijog.2020.v03i03.005

| Received: 18.02 .2020 | Accepted: 25.02 .2020 | Published: 21.03 .2020

*Corresponding author: Dr. R C Prameela

\section{Abstract}

Introduction: Over the last 15 years, maternal mortality has declined in most parts of the world. The maternal morbidity burden remains still high for which there is a need for greater awareness about the complications of childbirth. A better understanding helps in lessening the burden and helps in providing better services and to improve health of puerperal women. The aim of this study is to analyse various puerperal morbidities in women delivered at cheluvamba hospital, Mysuru. Method: This is a prospective descriptive observational study, done in cheluvamba hospital, attached to MMC\&RI, Mysuru, and Karnataka which is a tertiary care center. The study was done for a period of 12 months duration from november 2016 to october 2017. Parameters studied were age, parity, various puerperal morbidities, mode of delivery, onset of morbidity, risk factors. Results: During the study period, total number of deliveries in Cheluvamba hospital were 11327. cesarean sections were 3136 and total vaginal deliveries were 8191 .out of 11327 women,161 $(1.42 \%)$ women developed puerperal morbidities. the most common morbidity was wound infection and dehiscence seen in $46(28.6 \%)$ women, followed by breast complications seen in 39(24.2\%) women and puerperal pyrexia and respiratory tract infections in 20(12.4\%) each. Conclusion: The most common puerperal morbidity is wound related complications. The study showed that there was considerable puerperal morbidity after caesarean section. These complications can be reduced by designing and implementing evidence based and cost effective management guidelines.

Keywords: Puerperium; Morbidities; wound dehiscence; Risk factors.

Copyright @ 2020: This is an open-access article distributed under the terms of the Creative Commons Attribution license which permits unrestricted use, distribution, and reproduction in any medium for non-commercial use (NonCommercial, or CC-BY-NC) provided the original author and source are credited.

\section{INTRODUCTION}

Puerperal morbidities are a serious public health problem. The puerperal morbid consequences include puerperal fever and sepsis, anaemia, breast engorgement and abscess, episiotomy or caesarean section wound breakdown, perineal tears, urinary tract infection and urinary retention, and depression, thromboembolism, pneumonia; others defined in the literature as long-term morbidities include incontinence, fistula, pelvic inflammatory disease, genital prolapse, hypertension, haemorrhoids, nerve damage, pituitary failure, anaemia. Many of these arise during 1-2 weeks following delivery and can become chronic if not cared for appropriately.For each woman who dies due to complications of childbirth, $10-15$ others suffer from different forms of puerperal morbidities.Current data on the extent of puerperal morbidities in developing countries is extremely limited which do not permit accurate measurements of the incidence and prevalence of the full spectrum of puerperal morbidities.

\section{OBJECTIVES}

- To study the incidence of various puerperal morbidities in women who delivered at Cheluvamba hospital

- To Study the risk factors of puerperal morbidities.

\section{STUDY METHODS AND MATERIALS}

This is a prospective descriptive observational study, done in cheluvamba hospital, attached to MMC\&RI, Mysuru, and Karnataka which is a tertiary care center. The study was done for a period of 12 months duration from november 2016 to october 2017.All the women delivered at Cheluvamba Hospital who developed puerperal morbidities during their hospital stay or readmitted for puerperal morbidity were included and detailed medical history, reproductive 
history, general and systemic examination local examination was done and evaluated for different puerperal morbidities and managed accordingly. The women with puerperal morbidities delivered outside, women with previous medical disorder were excluded.

The results were analysed in terms of mode of delivery, age, parity, gestational age, different morbidity, onset of illness and risk factors. Data was then compiled on computer. The Statistical software namely SPSS 19.0 was used for the analysis of the data and Microsoft word and Excel have been used to generate graphs, tables etc. The results were analysed using following statistical methods: Descriptive statistical analysis has been carried out in the present study. Categorical measurements are presented in Number (\%).

\section{RESULTS}

Total number of deliveries in Cheluvamba hospital during the study period of 12 months were 11327 .The total number of caesarean sections were 3136 and total vaginal deliveries were 8191 .

Out of 11327 , total 161 women developed puerperal morbidities. The incidence of puerperal morbidities in our hospital was $1.42 \%$. Among 161 women, 58(36\%) women had vaginal delivery and $102(63.4 \%)$ had Caesarean section and 1 (0.6\%) had laparotomy and peripartum hysterectomy.

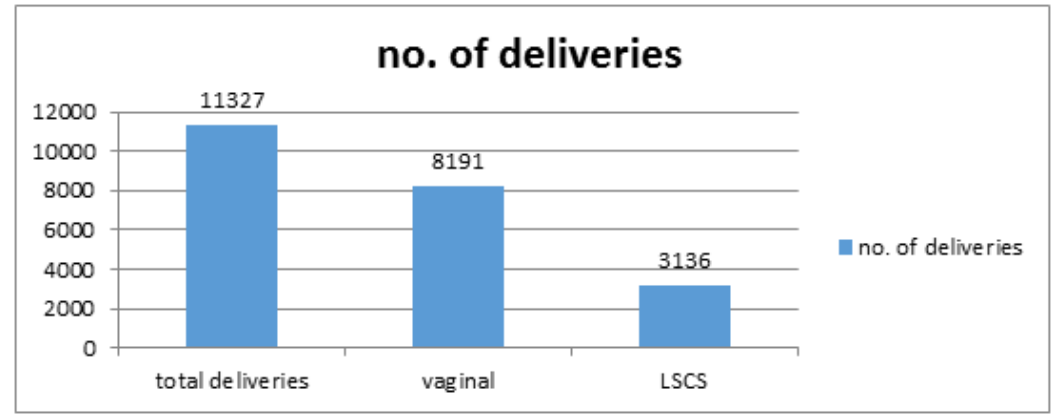

Among 8191 women who had vaginal deliveries, total $58(0.70 \%)$ had puerperal morbidities, whereas out of 3136 Caesarean section and laparotomy, $102(3.25 \%)$ and $1(0.03 \%)$ had morbidities, respectively. The incidence of postpartum morbidities in this study, in vaginal deliveries wad $0.70 \%$ and in Caesarean section was $3.25 \%$ and laparotomy was $0.03 \%$.

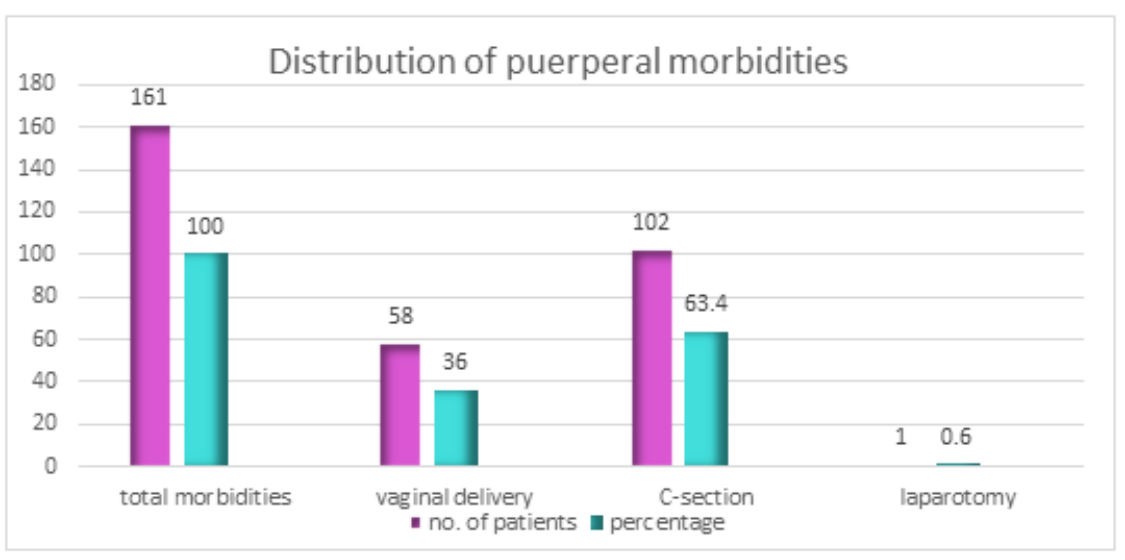

In this study, 35(21.7\%) women belonged to age $<20$ years, most women $114(70.8 \%)$ were between age 21 to 30 years, $11(6.8 \%)$ belonged to 31 to 40 years age group and only $1(0.6 \%)$ was above 41 years. The mean age among the study group was 23.99 years, minimum age being 17years and maximum age noted was 45years.100 (62.1\%) women were primipara,43(26.7\%) had parity 2 , while $16(9.9 \%)$ had parity 3 and $2(1.2 \%)$ had parity -4 .

In ANC care, 98(60.9\%) women were booked cases and $63(39.1 \%)$ were unbooked case. According to modified B.G.Prasad classification [1] classification of socioeconomic class, $120(74.5 \%)$ women belonged lower class, 21(13\%) belonged to upperlower class while $20(12.4 \%)$ belonged to uppermiddle class of socioeconomic status.7 (4.3\%) women had preterm delivery while $154(95.7 \%)$ women had term delivery among women who developed postnatal morbidities.

In our study, out of 161 women, 3(1.9\%) women had multiple gestation and $158(98.1 \%)$ had singleton pregnancy. 
Out of 161 women who developed postnatal morbidities, 102 (63.4\%) had Cesarean section, $58(36 \%)$ women had vaginal delivery and $1(0.6 \%)$ woman underwent laparotomy and peripartum hysterectomy. The most common morbidity found in our study was wound infections and dehiscence in 46 (28.6\%) women, followed by breast complications seen in $39(24.2 \%)$ women and puerperal pyrexia and respiratory tract infections in $20(12.4 \%$ ) each.8(5\%) women had UTI and secondary PPH each.6(3.7\%) women had postpartum eclampsia.4(2.5\%) had psychiatric complications.2(1.2\%) had blood transfusion reaction. other morbidities studied includedPPCM, DVT, neuropraxia of leg ,Bell's palsy, pubic symphysis separation, hemolytic anemia, vaginal wall hematoma and vesicovaginal fistula in $1(0.6 \%)$ each.

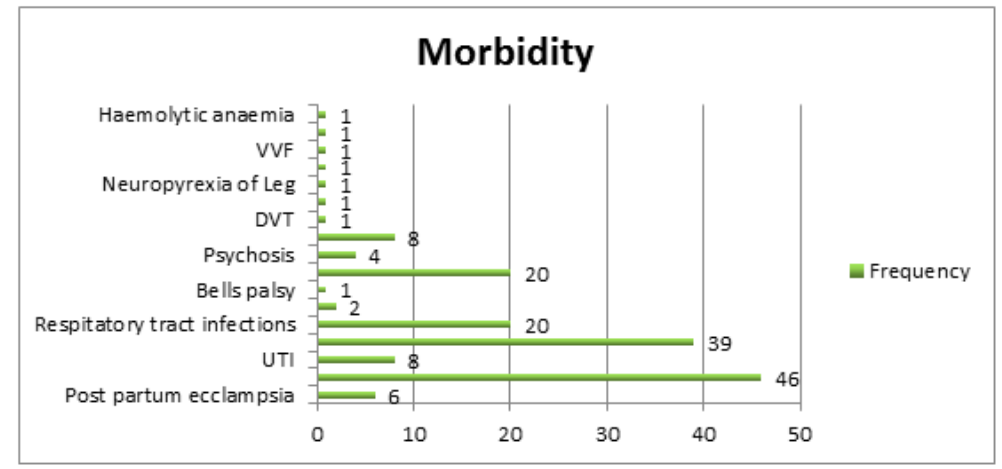

Most of the women $-131(81.4 \%)$ developed postnatal morbidity between 2 to 8 days after delivery.17 (10.6\%) developed from 9 to 15 days, $8(5 \%)$ developed on the first postnatal day, $2(1.2 \%)$ developed between 16 to 22 days and 2(1.2\%) developed between 23 to 29 days and 1(0.6\%) developed beyond 30days.

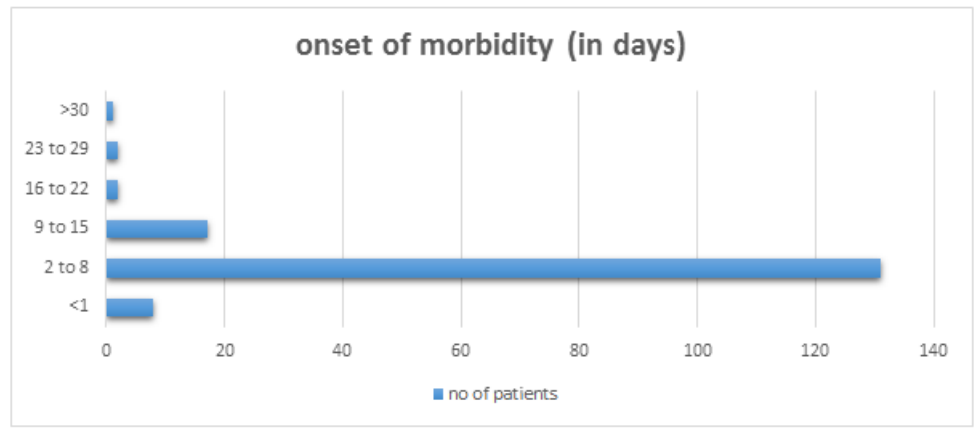

Among the various risk factors studied, most common risk factor was anaemia in 59(36.64\%), followed by preeclampsia in $48(29.81 \%)$ women and obesity in 24(14.90\%) women. Among anaemic women, $22(13.66 \%)$ had severe anemia and required blood and blood products transfusions. other risk factor studied was diabetes- found in $15(9.31 \%)$ women. Chorioamnionitis was seen in $18(11.18 \%)$ patients.

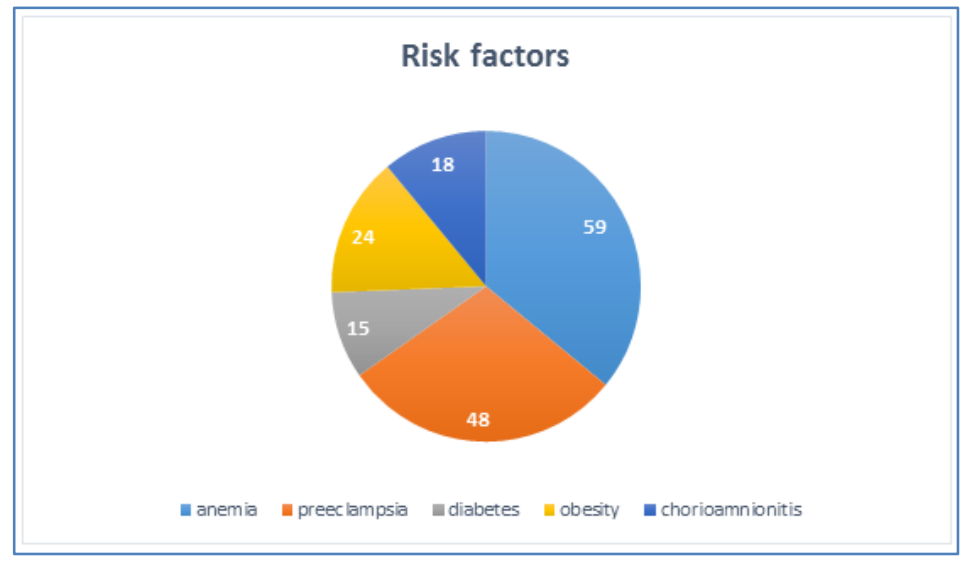


Among Culture from the discharge of wound, the most common organism isolated was Staph aureus in $11(27.5 \%)$ cases, followed by Aceinobacter species in $6(15 \%)$ cases. Pseudomonas and E.coli were isolated in $5(12.5 \%)$ cases each. Strep pyogenes obtained in $3(7.5 \%)$ cultures and Klebsiella in $2(5 \%)$, while no organism isolated in $8(20 \%)$ cases.

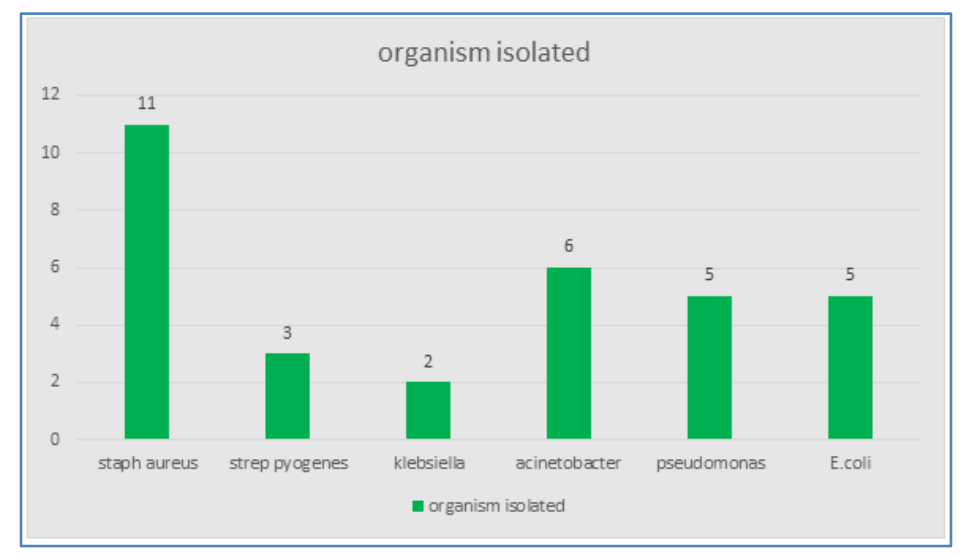

\section{DISCUSSION}

Postpartum period is a neglected part of maternal health. Consideration to improve maternal health during the postpartum period has to be done to improve the quality of life and to imbibe positive feelings towards the process of childbirth and the life afterwards. The physical as well as mental wellbeing of postpartum women are influenced by various factors. This study gives an insight into the burden of the different types of postnatal morbidities and their risk factors.

The incidence of puerperal morbidities was $1.4 \%$ in our study. In a cross sectional study done during august 2010 to december 2011 in urban slums of Jamnagar, Gujrath by Neha A. Patel et al. [2], 24.44\% women had morbidity during their post-partum period .In a cross sectional study by Navya Vyas et al. [3] during february to july 2013 done in Udupi, karnataka, postprtum morbidities were reported among $49 \%$ of the participants. In study conducted at Nepal by Rosy Malla and Reeta Manandhar [4], the incidence of postpartum morbidities was $1.58 \%$ which was comparable to our study.

In our study, majority $70.8 \%$ women belonged to the age group of 21 to 30years and the mean age was 23.99years. The mean age was 26.26 years in a cross sectional study by Navya Vyas et al. during february to july 2013 done in Udupi, karnataka.

Majority $(62.1 \%)$ of the women were primipara in our study.59\% were primiparas in study conducted in udupi, karnataka.

$26.1 \%$ women were illiterate, while $46 \%$ received primary education in our study population.in a study done in ramnagar, gujrath, $72 \%$ of the women did not have any formal education.study done in udupi revealed $52 \%$ women were illiterate and $38.9 \%$ had high school education among those developed postpartum morbidities.

Majority $74.5 \%$, belonged to lower socioeconomic class in our study which was comparable to study done in ramnagar where $>2 / 3^{\text {rd }}$ of the study population belonged to backward class and $72.45 \%$ were from lower socioeconomic class respectively.

In our study, $4.3 \%$ women among the study population had preterm delivery and $95.7 \%$ had term delivery. The mean gestational age studied in nepal study was 39-40 weeks both in vaginal delivery and Caesarean delivery group.in a study done in udupi, $68.6 \%$ women had full term delivery in the study population.

In our study, $36 \%$ had vaginal delivery and $63.4 \%$ had LSCS in study population. In a study done in Ghana [5] 45.6\% among caesarean delivery group developed morbidities.

In this study, wound infection and related complications was the most common morbidity $(28.6 \%)$, second most common was breast complications comprising $24.2 \%$ women and followed by puerperal pyrexia in $12.4 \%$ and respiratory tract infection in $12.4 \%$ women. Postpartum eclampsia was seen in $3.7 \%$ cases.

In our study most common organism isolated in puerperal sepsis cases was Staph aureus 30.76\%, followed by E.coli in $23.07 \%$ cases and Klebsiella in $15.38 \%$ cases. In a study by Sahiba $\mathrm{S}$ et al. [6], Out of 67 samples, $46(68.65 \%)$ yielded growth in blood culture. Gram negative organisms were isolated in 31 cases $(67.39 \%)$ while Gram positive organisms in 15 cases $(32.60 \%)$. Klebsiella was the most frequent 
isolate $13(28.26 \%)$, followed By Staph. aureus 10 $(21.73 \%)$.

\section{CONCLUSION}

The most common puerperal morbidity in the women delivered in cheluvamba hospital during the study period was -wound related complications. There is a need for improvement in care to reduce puerperal morbidity following caesarean section; like maintenance of strict asepsis,to evaluate the pattern and prevalence of microorganisms and their culture sensitivity pattern, to identify risk factors like anemia, diabetes, chorioamnionitis and to educate the puerperal women regarding hygeinic practices. The study showed that there was considerable puerperal morbidity after caesarean section.

\section{REFERENCES}

1. Shaikh, Z., \& Pathak, R. (2017). Revised Kuppuswamy and BG Prasad socio-economic scales for 2016. Int J Community Med Public Health, 4(4), 997-999.
2. Patel, N. A., Mehta, J. P., Unadkat, S., \& Yadav, S. B. (2016). A study on various determinants of maternal morbidity amongst married women in reproductive age group in urban slums of jamnagar, gujarat, india. Int J Cur Res Rev| Vol, 8(24), 19.

3. Vyas, N., Kamath, R., Pattanshetty, S., \& Binu, V. S. (2016). Postpartum related morbidities among women visiting government health facilities in Udupi Taluk, Karnataka, India. Journal of family medicine and primary care, 5(2), 320.

4. Malla, R., \& Manandhar, R. (2016). Puerperal Morbidities After Delivery in a Tertiary Care Referral Hospital of Nepal. Medical Journal of Shree Birendra Hospital, 15(2), 8-12.

5. Aduama, D. M., Obed, S. A., Seffah, J. D., \& Gumanga, S. K. (2015). Puerperal Morbidity Following Caesarean Section in a Teaching Hospital in Ghana. Journal of Gynecology and Obstetrics, 3(4), 92-97.

6. Tamboli, S. S., Tamboli, B. S., \& Shrikhande, S. (2017). Puerperal sepsis: predominant organisms and their antibiotic sensitivity pattern. Int J Reprod Contracept Obstet Gynecol, 5(3), 762-5. 